\title{
RESISTANCE OR NON-COMPLIANCE? ASSESSING THE ROLE OF LITERACY IN ADHERENCE TO MEDICATION
}

\author{
Vansh Chopra \\ Vasant Valley School
}

DOI: $10.46609 /$ IJSSER.2020.v05i04.013

URL:https://doi.org/10.46609/IJSSER.2020.v05i04.013

\begin{abstract}
Among the largest problems in the field of medicine is the issue of medical adherence. In both developed and developing countries, the non-adherence of patients causes recurrence in chronic illnesses, a strain on the public health system, and a general decline in economic and public health. This is especially so among the most vulnerable sections of the population, such as the elderly, women, minorities, and the poor. Several studies have concluded that there is a positive co-relation and a causal effect between literacy and medical adherence. It increases adherence to prescribed treatments when patients are made aware of the benefits of doing so, and the ill effects of non-adherence. Despite this finding, the field of healthcare policy continues to struggle with implementation of effective measures to tackle the same. This paper will examine the theoretical foundations of medical adherence, and the current policies that exist. The paper will then offer a critique of existing policy, and pose policy recommendations for a more holistic and multi-disciplinary approach that takes into account varying contexts and factors that interplay with literacy.
\end{abstract}

Keywords: Health literacy, Medical adherence, Healthcare, Medicine, Chronic illnesses

\section{INTRODUCTION}

Medical adherence is as important, if not more important, the diagnosis or prescription of medicines. Patients must adhere to the medication prescribed, as non-adherence carries with it several risks such as a resurgence of illness, or the patient developing a drug resistant form of the same illness. Adherence to taking medication is a catalyst to achieve prevention, cure, and wellbeing (Abderrahman, 2017). Patients require medications, and the majority understand that adherence is paramount. Physicians 'prescribe' the medications, yet medication adherence 


\section{International Journal of Social Science and Economic Research}

ISSN: $2455-8834$

Volume: 05, Issue: 04 "April 2020"

remains a major challenge. Further, it is a key factor of the therapy of chronic diseases in older people with chronic diseases (Yun-Mi Lee, 2017).

Adherence to long-term therapy for chronic illnesses in developed countries averages $50 \%$. In developing countries, the rates are even lower (WHO, 2003). It is undeniable that many patients experience difficulty in following treatment recommendations, in both developed and developing countries. Non-communicable diseases and mental disorders, human immunodeficiency virus/acquired immunodeficiency syndrome and tuberculosis, together represented $54 \%$ of the burden of all diseases worldwide in 2001 and will exceed 65\% worldwide in 2020 (WHO, 2003) The poor are disproportionately affected. In developed countries, the epidemiological shift in disease burden from acute to chronic diseases over the past 50 years has rendered acute care models of health service delivery inadequate to address the health needs of the population (WHO, 2003) . In developing countries, this shift is occurring at a much faster rate (Omotosho and Ayegha, 2019).

Therefore, paying attention to policy surrounding medical adherence is crucial. There is a linkage and causal relationship between literacy and medical adherence. When patients are not adequately informed of its importance, they tend to disregard the need for continuance with long term medication (Miller, 2016). Especially in developing nations and societies which are religious or not familiar with Western medicine, this increases reliance on traditional or alternative means of treatment. Poor adherence to long-term therapies severely compromises the effectiveness of treatment making this a critical issue in population health both from the perspective of quality of life and of health economics (WHO, 2003). Interventions aimed at improving adherence would provide a significant positive return on investment through primary prevention of risk factors and secondary prevention of adverse health outcomes (WHO, 2003).

This paper will examine the theories and components of medical adherence, with a review of studies and research that indicates a link between literacy and medical adherence in different contexts. The paper will then pose policy recommendations for the improvement of literacy on adherence in different contexts, and the consequent improvement of the public health system.

\section{BACKGROUND}

Over time, the literature on medical adherence has created a distinction between compliance and adherence. Previously, the term compliance was widely used to describe how patients comply with their physician's wishes regarding a remedy. It was criticised because of its paternalistic nature, directed from the physician towards the patient (Adberrahman, 2017). The definition of adherence thus came to be a measure of how persistent patients are in taking their medications (Abderrahman, 2017). The adherence project has adopted the following definition of adherence 


\section{International Journal of Social Science and Economic Research}

ISSN: $2455-8834$

Volume: 05, Issue: 04 "April 2020"

to long-term therapy: the extent to which a person's behaviour - taking medication, following a diet, and/or executing lifestyle changes, corresponds with agreed recommendations from a health care provider (WHO, 2003).

Non-adherence can be categorized into three broad types. Firstly, Non-adherence is a situation when patients never fill or initiate their medications and this is divided into intentional, which is determined by the patient's beliefs, expectations and attitudes; and unintentional, which is determined by limitations of resources and personal constraints (Abderrahman, 2017). Nonpersistence is when patients stop taking the medication after starting it (Adberrahman, 2017). Non-conforming is a situation when patients do not take the medication properly as described, whether it is the dose or time, among other factors (Jimmy, 2011). Factors influencing adherence vary from social and economic-related, such as poverty, medication costs and insufficient insurance coverage, to patient-related factors including forgetfulness and health misconceptions, medication-related factors like the number of medications, medication dosage and frequency (Osterberg, 2005).

There has been a great deal of secondary study linking literacy and medical adherence in several differing contexts. Some have observed that employment status and higher education exerted a positive effect on adherence (Mathes et al, 2014). Some have found that communication by the physician is a key factor to improve literacy (Lehman et al, 1997; Abderrahman, 2017). Adherence problems are observed in all situations where the self-administration of treatment is required, regardless of type of disease, disease severity and accessibility to health resources (WHO, 2003). This has been observed across several diseases, such as asthma, diabetes, hypertension and HIV/AIDS. To ascertain the true extent of adherence, data on developing countries and important subgroups, such as adolescents, children and marginal populations are urgently required (WHO, 2003). A full picture of the magnitude of the problem is critical to developing effective policy support for efforts aimed at improving adherence. In the following section, secondary studies will be discussed in further detail, as well as an evaluation of programmes implemented globally to ensure medical adherence.

\section{DISCUSSION}

In the global context, there is no single intervention strategy, or package of strategies that has been shown to be effective across all patients, conditions and settings (WHO, 2003). Consequently, interventions that target adherence must be tailored to the particular illness-related demands experienced by the patient. To accomplish this, health systems and providers need to develop means of accurately assessing not only adherence, but also those factors that influence it (WHO, 2003). Current strategies that are most widely followed and implemented are education 


\section{International Journal of Social Science and Economic Research}

ISSN: $2455-8834$

Volume: 05, Issue: 04 "April 2020"

in self-management, pharmacy management programmes, nurse, pharmacist and other nonmedical health professional intervention protocols, counselling, behavioural interventions, follow-up and reminders, among others (WHO, 2003). However, it has been observed that these policies have tended to be used alone (WHO, 2003). A single-factor approach might be expected to have limited effectiveness, if the factors determining adherence interact and potentiate each other's influence as they are likely to do (WHO, 2003). The most effective approaches have been shown to be multi-level - targeting more than one factor with more than one intervention (Woller et al, 1993; Wagner et al, 2001)). Several programmes have demonstrated good results using multilevel team approaches. Adequate evidence exists to support the use of innovative, modified health care system teams rather than traditional, independent physician practice and minimally structured systems (DeBusk et al, 1994; Peters et al, 1995).

The most vulnerable populations in this respect are the elderly with chronic illnesses, the poor and those who are either illiterate or have poor literacy. Studies have shown that patients with low literacy may have initial difficulty with learning self-care behaviors and enacting them, but that with ongoing support and additional training, they can overcome these barriers (Pignone and DeWalt, 2006). There have also been studies focused on the elderly, who are most susceptible to long term, chronic illnesses. Studies in Korea showed that implementation and evaluation of health literacy interventions for older people with chronic conditions are important to increase medication adherence and potentially improve patient outcomes (Yun-Mi Lee et al, 2017).

However, it also important to note that other factors go hand in hand with literacy. Therefore, it cannot be approached as a singular issue. Poor literacy, in both developed and developing countries is mostly a consequence of systemic barriers which require structural reform (WHO, 2003). Therefore, along with interventions that are tailored to individual patients or a group, policy makers must approach the issue from a more structural point of view to take into account the varying contexts. For example, structural reform in the insurance and healthcare industry itself is required. Studies have found that co-payments, higher medication cost, medication regimen complexity, and the status of ethnic minorities exerted a negative effect, which all point to more deep seated issues that consequently have an impact on literacy (Mathes et al, 2014).

Few studies have evaluated programmes that have used such interventions, and this is a serious gap in the applied knowledge base. For an intervention to be truly multi-level, systemic barriers must be included (WHO, 2003). Unless variables such as these are addressed, it would be expected that the impact of the efforts of providers and patients would be limited by the external constraints (WHO, 2003). The problem of non-adherence has been much discussed, but has been relatively neglected in the mainstream delivery of primary care health services. Despite an extensive knowledge base, efforts to address the problem have been fragmented, and with few 


\section{International Journal of Social Science and Economic Research}

ISSN: $2455-8834$

Volume: 05, Issue: 04 "April 2020"

exceptions have failed to harness the potential contributions of the diverse health disciplines (WHO, 2003). A stronger commitment to a multidisciplinary approach is needed in order to make progress in this area. This will require coordinated action from health professionals, researchers, health planners and policy-makers (WHO, 2003). In the following concluding section, this paper will pose policy recommendations for a more multidisciplinary approach to healthcare literacy and intervention.

\section{CONCLUSION}

It is clear that in order for effective change in the realm of healthcare policy, health literacy must be viewed as a multi-faceted model (Adberrahman, 2017). It must be functional, which reflects the outcomes of traditional education on health risk and how to use health systems (Abderrahman, 2017). It should be interactive, with a focus on creating a supportive environment to patients and how they can acquire social skills towards a healthier life. It must also be critical, which means patients should be able to analyse health information more efficiently and have a better control over their health (Abderrahman, 2017). To better improve literacy and medical adherence, a more holistic approach is required which is tackled from several angles and not only centered on the patient and physician.

There must be social and economic interventions, in the realms of poverty, access to health care and medicines, illiteracy, provision of effective social support networks and mechanisms for the delivery of health services that are sensitive to cultural beliefs about illness and treatment (Garnett, 2000; WHO, 2003). Community-based organizations, education of illiterate patients, assessment of social needs and family preparedness have been reported to be effective social interventions for improving adherence (Ruggieron, et al, 1990; Glasgow et al, 1987).

At the level of prescribing, physicians are advised to simplify the medication taking, and involve patients in the treatment plan (Abderrahman, 2017). Healthcare system interventions must involve extensive training of front line staff, and must go beyond the provision of advice and prescriptions. If either the perceived value of adhering, or confidence, is low, the likelihood of adherence will also be low (WHO, 2003). Health care providers can learn to assess the potential for non-adherence, and to detect non-adherence itself. They can then use this information to implement brief interventions to encourage and support progress towards adherence (WHO, 2003). A conceptual framework that explains how patients progress to adherence will help practitioners to tailor their interventions to the needs of the patient. As a consequence, literacy of the patient is greatly impacted (WHO, 2003).

Written instructions about medication that are culturally appropriate and adapted to suit the patient's level of literacy should be a core part of every interaction with the patient (WHO, 


\section{International Journal of Social Science and Economic Research}

ISSN: $2455-8834$

Volume: 05, Issue: 04 "April 2020"

2003). For example, studies on medication regimens for asthmatic older patients found that comprehension and recall of information on how to take medication was shown to be significantly improved when medication-taking instructions were clear, presented as lists rather than paragraphs, used pictures or icons in combination with written medication instructions and were consistent with patients' mental representations of medication taking (Morrow et al, 1998).

Women and minorities have also found to lack literacy skills in several societies, more than men. Women may also in several societies may not have as much decision making power with respect to their own health issues, which negatively impacts their medical adherence. Therefore, intervention and awareness programs targeted specifically towards women and minorities would be required. Future research in developing countries should consider individual risk perceptions, cultural barriers, gender and the role of local health system in health care delivery (Dhar et al, 2017).

Studies consistently find significant cost-savings and increases in the effectiveness of health interventions that are attributable to low-cost interventions for improving adherence (WHO, 2003). Without a system that addresses the determinants of adherence, advances in biomedical technology will fail to realize their potential to reduce the burden of chronic illness. Access to medications is necessary but insufficient in itself for the successful treatment of disease (WHO, 2003). A burgeoning area of study is the use of technology and ICT in tackling medical adherence issues (Omotosho and Ayega, 2019). Research suggests that low-income countries can adapt some of these technologies into managing patient's adherence, starting with health education of patients', breaking the barriers created by proximity, and cost of visits hospital visits, recovery from many of the prevalent diseases can be improved through electronic prescription and mobile reminders (Omotosho and Ayega, 2019). Therefore, it is clear that increasing literacy is the main policy option to target a wide range of issues. It is a gateway any further policy interventions.

\section{REFERENCES}

Abderrahman, B., (November 2017), "Health literacy, medication adherence and thriving healthcare systems: connecting the dots", The Pharmaceutical Journal, Retrieved from https://www.pharmaceutical-journal.com/opinion/insight/health-literacy-medicationadherence-and-thriving-healthcare-systems-connecting-thedots/20203831.article?firstPass=false\#fn_13

DeBusk RF et al. (1994), “A case-management system for coronary risk factor modification after acute myocardial infarction”, Annals of Internal Medicine, 120:721-729 
International Journal of Social Science and Economic Research

ISSN: $2455-8834$

Volume: 05, Issue: 04 "April 2020"

Dhar, L., et al, (2017), "A Systematic Review of Factors Influencing Medication Adherence to Hypertension Treatment in Developing Countries", Open Journal of Epedimiology, Vol. 7, Issue 3

Garnett WR. (2000), "Antiepileptic drug treatment: outcomes and adherence", Pharmacotherapy, 20:191S-199S.

Glasgow RE, McCaul KD, Schafer LC. (1987), "Self care behaviors and glycemic control in Type 1 diabetes", Journal of Chronic Diseases, 40:399-412.

Jimmy B \& Jose J, (2011), "Patient medication adherence: measures in daily practice.", Oman Medical Journal, 26(3):155-159.

Lehmann LS, Brancati FL, Chen MC et al., (1997), "The effect of bedside case presentations on patients' perceptions of their medical care", New England Journal of Medicine, 336(16):1150—1155

Mathes T, Jaschinski T \& Pieper D. (2014), "Adherence influencing factors - a systematic review of systematic reviews", Arch Public Health, 72(1):37

Miller, T., (2016), "Health Literacy and Adherence to Medical Treatment in Chronic and Acute Illness: A Meta-Analysis", Patient Education Counselling, Vol. 99 Issue 7, pp 1079-1086

Morrow DG et al, (1998), "The influence of list format and category headers on age differences in understanding medication instructions". Experimental Aging Research, 24:231-256

Omotosho, A and Ayegha, P, (July 2019), "Medication Adherence A Review and Lessons for Developing Countries", International Journal of Online Engineering

Osterberg L \& Blaschke T, (2005), "Adherence to medication", New England Journal of Medicine, 353(5):487-497

Peters AL, Davidson MB, (1995), Ossorio RC, "Management of patients with diabetes by nurses with support of subspecialists”, HMO Practice, 9:8-13

Pignone, M, and DeWalt, D., (2006), "Literacy and Health Outcomes: Is Adherence the Missing Link?”, Journal of General International Medicine, Vol. 21 Issue 8, pp 896-897

Ruggieron L et al, (1990), "Impact of social support and stress on compliance in women with gestational diabetes", Diabetes Care, 13:441-443. 
Wagner EH et al, (2001), "Chronic care clinics for diabetes in primary care: A system-wide randomized trial", Diabetes Care, 24:695-700.

Woller W et al., (1993), "Cortisone image and emotional support by key figures in patients with bronchial asthma: An empirical study”, Psychotherapy \& Psychosomatics, 59:190-196.

World Health Organization, (2003), “Adherence to Long Term Therapies: Evidence for action”, Retrieved

from https://www.who.int/chp/knowledge/publications/adherence_full_report.pdf

Yun, Mi-Lee, et al, (2017), "Impact of health literacy on medication adherence in older people with chronic diseases", Collegian, Vol. 24 Issue 1 